study of patients who were entered into the National Asthma Database following investigations under the Difficult Asthma Protocol (DAP) between 2007 and 2011. The following were included: Age at diagnosis, liver function (at initial assessment and most recent), liver imaging, glucose and lipids at initial investigation, and medication history.

Results 209 subjects were included in the audit, all of whom entered into the National Asthma Database between 2007 and 2011. Mean age was 45 at presentation to $\mathrm{RBH}$.

Abnormal Liver Function $20 \%(n=41)$ patients had abnormal liver function tests either at first presentation to $\mathrm{RBH}$ or on their most recent blood tests. Only 14 (34\%) were further investigated with liver imaging. Of those with deranged liver function who were imaged, $79 \%(n=11)$ had radiological evidence of NAFLD.

Management of patients with confirmed NAFLD Of the patients with confirmed NAFLD only $27 \%(n=3)$ had their fasting lipids and glucose measured. $18 \%(n=2)$ were prescribed a statin and a similar number were prescribed metformin.

Asthma medications of patients subsequently diagnosed with NAFLD $81 \%$ of patients were prescribed aminophylline. $54 \%$ were prescribed montelukast and $81 \%$ patients were prescribed oral corticosteroids. $36 \%$ of patients were treated with omalizumab (anti-IgE).

Conclusion Amongst our cohort of wevere asthmatics a substantial proportion have deranged liver function. Of those with abnormal LFT who underwent imaging, a high proportion (79\%) had radiological evidence of NAFLD. However $66 \%$ of patients with deranged LFT did not proceed to liver imaging. This suggests that NAFLD is currently under diagnosed and managed in this patient population

Disclosure of Interest None Declared

\section{PTU-108 AZATHIOPRINE PHARMACOGENETICS IN AUTOIMIMUNE HEPATITIS}

doi:10.1136/gutjnl-2013-304907.198

1.2, ${ }^{*}$ Dhaliwal, ${ }^{2} \mathrm{E}$ McFarlane, ${ }^{2} \mathrm{D}$ Gleeson, 'L Lennard. 'Human Metabolism, University of Sheffield; 'iver Unit, Royal Hallamshire Hospital, Sheffield, UK

Introduction Azathioprine (AZA) is widely used to treat autoimmune hepatitis (AIH). However, $20 \%$ of patients are intolerant of AZA and a further $18 \%$ are unresponsive. AZA metabolism is complex and in childhood leukaemia, genetic polymorphisms in thiopurine methyltransferase (TPMT) and inosine triphosphate pyrophosphatase (ITPA) have been associated with AZA toxicity and differences in active metabolite production. This has been little studied in AIH. We aimed to assess the association of these polymorphisms with AZA toxicity and with accumulation of AZA derived thioguanine nucleotide (TGN) and methylmercaptopurine metabolites (MeMPNs) in AIH.

Methods We studied 151 patients with AIH (123 female; median age at diagnosis 55 (range 2-81) years). Subjects were genotyped for the presence of TPMT ${ }^{*} 3, T P M T{ }^{*} 2$ and ITPA $(94 \mathrm{C}>\mathrm{A}$ and IVS2 $+21 \mathrm{~A}>\mathrm{C}$ ) variant alleles. TGNs and and MeMPNs were measured in patients who remained on AZA.

Results For TPMT, 138 patients were wildtype and 13 (9\%) were heterozygous (1 TPMT ${ }^{*} 2,11 T P M T^{*} 3 A$ and 1 TPMT ${ }^{*} 3 C$ ). For ITPA, 95 were wildtype, 50(33\%) heterozygous (10 94C > A and 40 IVS2 $+21 \mathrm{~A}>\mathrm{C}$ ) and 6 homozygous/compound heterozygous. There were 57 adverse events (AE) in 54(36\%) patients - in 32 (21\%) AZA was withdrawn. TPMT wildtype and heterozygous patients had a similar incidence of leucopenia (18 vs $17 \%, p=0.9$ ) and of nonmyelotoxic AEs (21 vs $8 \% \mathrm{p}=0.5$ ). Likewise, ITPA wildtype, heterozygous and homozygous patients had a similar incidence of leucopenia $(19,10$ and $16 \%$ respectively, $p=0.2)$ and of non-myelotoxic AEs (20, 39 and $15 \%, p=0.2)$. Compared to wildtype patients, TPMT heterozygotes accumulated higher concentrations of TGNs (413 vs $\left.212 \mathrm{pmol} / 8 \times 10^{8} \mathrm{RBCs}, \mathrm{p}=0.009\right)$ and lower MeMPNs (111 vs. $\left.1000 \mathrm{pmol} / 8 \times 10^{8} \mathrm{RBCs}, \mathrm{p}<0.001\right)$, despite being a lower doses of AZA (1.0 vs. $1.7 \mathrm{mg} / \mathrm{kg} /$ day, p < 0.001). Comparing IPTA wildtype, heterozygous and homozygous patients, there was no difference in TGNs $\left(222,212\right.$ and $176 \mathrm{pmol} / 8 \times 10^{8} \mathrm{RBCs}$ respectively, $\mathrm{p}=0.76$ ) and MeMPNs (957, 957 and $713 \mathrm{pmol} / 8 \times 10^{8} \mathrm{RBCs}$ respectively, $\mathrm{p}=0.7$ )

Conclusion TPMT and ITPA polymorphisms do not predict the occurrence of adverse events in AIH. However, TPMT genotyping may be clinically useful as heterozygous patients may require a lower dose of AZA.

Disclosure of Interest None Declared

\section{PTU-109 PLASMA HEAT SHOCK PROTEIN-32 LEVELS IN PATIENTS WITH HEPATITIS C VIRUS-RELATED CIRRHOSIS: RELATION TO RENAL FUNCTION AND HEMODYNAMICS}

doi:10.1136/gutjnl-2013-304907.199

1, ${ }^{*} \mathrm{H}$ A El Aggan, ${ }^{2} \mathrm{M}$ I Reda, ${ }^{3} \mathrm{M}$ M Rizk. 'Department of Medicine; ${ }^{2}$ Department of Radiodiagnosis; ${ }^{3}$ Department of Clinical Pathology, Faculty of Medicine, University of Alexandria, Alexandria, Egypt

Introduction Heat shock protein-32 (HSP-32) is a microsomal enzyme that has hemodynamic effects and may play a role in the pathogenesis of renal diseases. Therefore, the present work was designed to study the plasma levels of HSP-32 and its product carbon monoxide (CO) in patients with hepatitis $\mathrm{C}$ virus (HCV)related cirrhosis in relation to renal function and hemodynamics. Methods Thirty patients with HCV-related cirrhosis and 15 healthy subjects were included in the study. The severity of liver disease was assessed using Child-Pugh classification and The Model for End-Stage Liver Disease (MELD) score. Renal function was evaluated by serum creatinine $(\mathrm{sCr}$ ) level, estimated glomerular filtration rate (eGFR) and urine sodium (UNa)concentration. Plasma HSP-32 levels were measured using commercially available enzyme-linked immunosorbant assay. Blood carboxyhemoglobin (COHB) concentration, an index of $\mathrm{CO}$ production, was assayed by spectrophotometry. Renal hemodynamics including renal artery peak systolic velocity (PSV), end-diastolic velocity (EDV), mean velocity $(\mathrm{MnV})$, resistive index $(\mathrm{RI})$ and pulsatility index (PI) and renal blood flow (RBF), were measured using Doppler ultrasonography.

Results Patients with HCV-related cirrhosis showed significant increases in plasma HSP-32 levels, blood COHB concentration and renal artery RI and PI and significant decreases in RBF and renal artery EDV and $\mathrm{MnV}$ compared with healthy subjects $(P<$ 0.001) The plasma HSP-32 levels and blood COHB concentration showed positive correlations with Child-Pugh and MELD scores, $\mathrm{sCr}$ and renal artery RI and PI and negative correlations with eGFR, UNa concentration, RBF and renal artery EDV and $\mathrm{MnV}(P$ $<0.05$ ). No correlations were found between plasma HSP-32 levels and blood COHB concentration on one hand and age of the patient, apparent duration of $\mathrm{HCV}$ infection and serum HCV RNA levels on the other hand $(P>0.05)$.

Conclusion The increased HSP-32 activity with enhanced endogenous $\mathrm{CO}$ generation may play an important role in renal dysfunction in HCV-related cirrhosis and could be a potential therapeutic target.

Disclosure of Interest None Declared 